\title{
Comparative efficacy of glimepiride and metformin in monotherapy of type 2 diabetes mellitus: meta-analysis of randomized controlled trials
}

Hongmei Zhu' ${ }^{1}$, Shuang Zhu' ${ }^{1}$ Xiuqian Zhang ${ }^{1}$, Yang Guo ${ }^{1}$, Yunzhen Shi ${ }^{1}$, Zhimin Chen ${ }^{1}$ and Siu-wai Leung ${ }^{1,2^{*}}$

\begin{abstract}
Background: Metformin treatment has been the most recommended monotherapy of type 2 diabetes mellitus (T2DM) for decades but is challenged by new antidiabetic drugs. This study conducted a meta-analysis of randomized controlled trials (RCT) comparing the efficacy of metformin and glimepiride in monotherapy of T2DM.

Methods: A literature search for RCTs on glimepiride and metformin was conducted on the bibliographic databases, including PubMed, Cochrane Library and ScienceDirect, from their inceptions to 25 Mar 2013. All RCTs were selected according to pre-specified eligibility criteria. The quality of articles was assessed with the Cochrane's risk of bias tool. Statistical meta-analysis evaluated the overall effects and biochemical indices of T2DM. Sensitivity and subgroup analyses evaluated the robustness and explained the heterogeneity of the results. Begg and Egger's tests quantified possible publication biases. Results were represented as "standard mean difference or odds ratio [95\% confidence internals] P value".

Results: Fifteen RCTs with 1681 adult T2DM patients were included for meta-analysis. Metformin was not better than glimepiride in overall efficacy in controlling the levels of HbA1c, postprandial blood sugar (PPBS), fasting plasma insulin (FINS), systolic and diastolic blood pressures (SBP and DBP), and high density lipoprotein (HDL). Metformin was only more effective than glimepiride in controlling the levels of total cholesterol (TC, 0.33 [0.03, $0.63], P=0.03)$, low-density lipoprotein $(\mathrm{LDL}, 0.35[0.16,0.53], P=0.0002)$ and triglycerides $(\mathrm{TG}, 0.26[0.05,0.46]$, $P=0.01$ ). Odds ratios of adverse events showed that glimepiride was more likely to induce hypoglycemia episodes and metformin was with a higher risk of gastrointestinal upset.
\end{abstract}

Conclusion: Metformin was not significantly better than glimepiride in glycemic control of T2DM, suggesting that glimepiride would be a good choice second to metformin in the monotherapy of T2DM.

Keywords: Glimepiride, Metformin, Type 2 diabetes mellitus, Meta-analysis

\section{Introduction}

Metformin has been the most recommended monotherapy of type 2 diabetes mellitus (T2DM) [1,2]. The UK Prospective Diabetes Study (UKPDS) found metformin more effective than chlorpropamide, glibenclamide and insulin [3-5]. The American Diabetes Association (ADA) recommended metformin as the first drug of choice for

\footnotetext{
* Correspondence: siu@inf.ed.ac.uk

${ }^{1}$ State Key Laboratory of Quality Research in Chinese Medicine, Institute of Chinese Medical Sciences, University of Macau, Macao, China

${ }^{2}$ School of Informatics, University of Edinburgh, Edinburgh EH8 9AB, UK
}

treating T2DM patients, especially those who are overweight [2]. The UK National Institute for Health and Clinical Excellence (NICE) recommended metformin if the patients are at danger under hypoglycaemia [1]. The latest recommendations of ADA [2] and NICE [1] were updated with the results of UKPDS [6,7], post-trial monitoring of UKPDS [8], and systematic reviews of comparing metformin with placebo, sulfonylureas and other antidiabetic drugs $[7,9]$, as well as the randomized controlled trials (RCTs) comparing metformin monotherapy with pioglitazone [10], metformin plus nateglinide [11], metformin 
plus rosiglitazone [12] and other non-metformin treatments [13]. A meta-analysis of RCTs on the efficacy of metformin in treating T2DM [14] found metformin lacking clear evidence for efficacy over the conventional or placebo treatment. A recent literature review suggested that metformin, albeit old, remained the best treatment for T2DM [15] but the review was not a systematic review or meta-analysis. It did not include the latest RCTs comparing metformin and glimepiride in monotherapy of T2DM.
Glimepiride is of the latest generation sulfonylureas for treating T2DM [16]. It has a lower cardiovascular risk than conventional sulfonylureas do [17-19]. Recent RCTs found it comparable to metformin in treating T2DM patients $[20,21]$ including those who are not responding well to non-glimepiride sulfonylureas [22,23]. Probably due to the late launch of glimepiride $[24,25]$ and lack of head-to-head comparative RCTs, early UKPDS, ADA and NICE's recommendations did not include the results of RCTs comparing metformin with

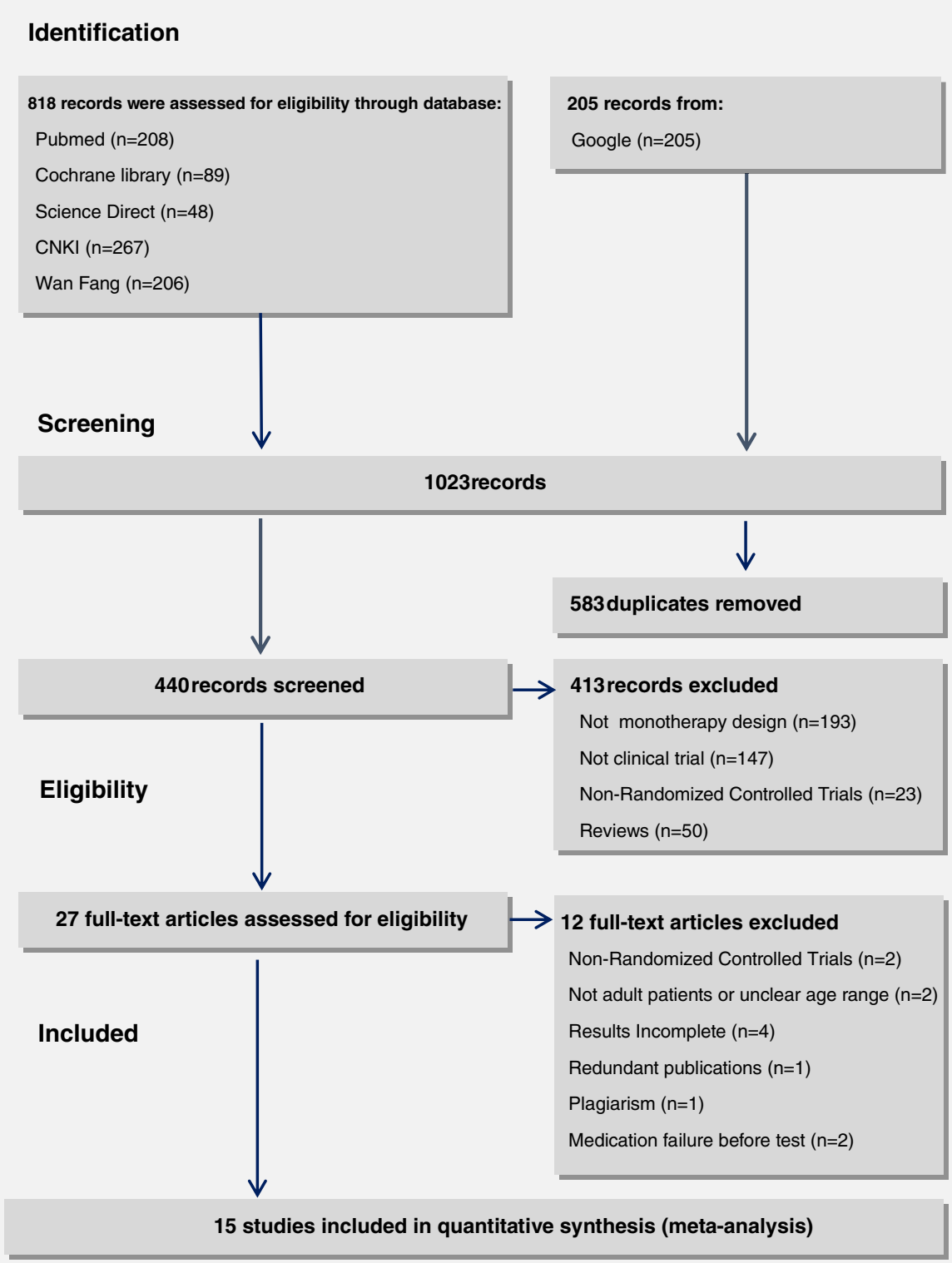

Figure 1 Flow of study selection. 
Table 1 Characteristics of included studies

\begin{tabular}{|c|c|c|c|c|c|c|c|c|}
\hline \multirow[t]{2}{*}{ Author (year) } & \multirow[t]{2}{*}{ Country } & \multirow[t]{2}{*}{ Age* $^{*}$} & \multicolumn{2}{|c|}{ Sample size } & \multicolumn{2}{|r|}{ Dosage } & \multirow{2}{*}{$\begin{array}{c}\text { Follow-up } \\
\text { period (weeks) }\end{array}$} & \multirow[b]{2}{*}{ Ref } \\
\hline & & & Glimepiride & Metformin & Glimepiride & Metformin & & \\
\hline \multirow[t]{2}{*}{ Ling (2003) } & China & Female 54 (8.3) & 50 & 44 & $1-6 \mathrm{mg} /$ day & 750-1750 mg/day & 12 & {$[33]$} \\
\hline & & Male 53 (9.2) & & & & & & \\
\hline Ramachandran (2004) & India & $30-60$ & 18 & 21 & $1-2 \mathrm{mg} /$ day & 250-850 mg/day & 14 & [34] \\
\hline Derosa (2004) & Italian & $46-67$ & 81 & 83 & 2-4 mg/day & 2000-3000 mg/day & 52 & [35] \\
\hline Gonzolez (2004) & Mexico & $40-65$ & 37 & 33 & $2 \mathrm{mg} /$ day & 2000 mg/day & 12 & [36] \\
\hline Rong (2004) & China & $42(11)$ & 98 & 100 & 4 mg/day & 1500 mg/day & 12 & {$[22]$} \\
\hline Tang (2004) & China & $35-70$ & 33 & 29 & 1-2 mg/day & 750-1500 mg/day & 26 & {$[37]$} \\
\hline \multirow[t]{2}{*}{ Yamanouchi (2005) } & Japan & Metformin 54.7 (9.8) & 37 & 39 & $1-2 \mathrm{mg} /$ day & 750 mg/day & 52 & {$[20]$} \\
\hline & & Glimepiride 53 (9.2) & & & & & & \\
\hline Ning (2006) & China & $35-70$ & 51 & 50 & $1-6$ mg/day & $250 \mathrm{mg}$ bid-750 mg tid & 52 & [38] \\
\hline Wu (2007) & China & $30-70$ & 40 & 30 & $1-6$ mg/day & $250-2000 \mathrm{mg}$ tid & 12 & [39] \\
\hline Xu (2007) & China & $35-70$ & 34 & 34 & $1-6 \mathrm{mg} /$ day & $250 \mathrm{mg}$ to maximum bid & - & {$[40]$} \\
\hline Li (2007) & China & $32-70$ & 35 & 33 & 2-6 mg/day & $250-1500 \mathrm{mg}$ bid & 65 & [41] \\
\hline Wang (2009) & China & $45(7)$ & 49 & 50 & 4-6 mg/day & 1500 mg/day & 12 & [23] \\
\hline \multirow[t]{2}{*}{ Rahman (2011) } & Pakistan & Metformin 51.9 (14.1) & 102 & 102 & 2-8 mg/day & $500-2000 \mathrm{mg} / \mathrm{day}$ & 52 & [42] \\
\hline & & Glimepiride 52 (15.4) & & & & & & \\
\hline Yoon (2011) & Korea & $30-65$ & 118 & 114 & 2 mg/day & 500 mg/day & 48 & [21] \\
\hline Wang (2011) & China & $33-70$ & 68 & 68 & $2 \mathrm{mg} /$ day & $500 \mathrm{mg}$ bid & 12 & {$[43]$} \\
\hline
\end{tabular}

* presented in range or mean (standard deviation).

glimepiride in monotherapy but they did include the findings that sulfonlyureas had increased risks in hypoglycemia, weight gain and cardiovascular issues. Recent cohort studies confirmed the increased cardiovascular risks of glimepiride [26] but did no cardiovascular harm to the patients with diagnosed coronary artery disease [27]. This study aimed to compare the efficacy between metformin and glimepiride in monotherapy of T2DM through a meta-analysis and supply the evidence that was missing from previous reviews $[9,11]$ and clinical guidelines $[1,2]$.

\section{Methods}

\section{Eligibility criteria}

\section{Inclusion criteria}

This study included the RCTs comparing glimepiride with metformin as monotherapy of T2DM. Participants in the RCTs were adult patients suffering from T2DM. Outcome measures of the treatment of T2DM included BMI (body mass index), SBP (systolic blood pressure), DBP (diastolic blood pressure), FPG (fasting plasma glucose), HbA1c (glycosylated hemoglobin level), PPBS (postprandial blood sugar), TC (total cholesterol), HDL

Table 2 Results of overall efficacy

\begin{tabular}{|c|c|c|c|c|c|c|c|}
\hline \multirow[t]{2}{*}{ Outcome } & \multirow{2}{*}{$\begin{array}{l}\text { No. of } \\
\text { studies }\end{array}$} & \multirow[t]{2}{*}{ Pooled sample size } & \multicolumn{3}{|c|}{ Heterogeneity } & \multicolumn{2}{|c|}{ Overall effect } \\
\hline & & & $\overline{T^{2}}$ & $1^{2}$ & P-value & SMD [95\% Cl] & P-value \\
\hline$\overline{B M I}$ & 10 & 988 & 0.50 & $92 \%$ & $<0.00001$ & $-0.06[-0.53,0.40]$ & 0.79 \\
\hline SBP & 5 & 615 & 0.22 & $86 \%$ & $<0.0001$ & $0.39[-0.06,0.83]$ & 0.09 \\
\hline DBP & 5 & 615 & 0.23 & $87 \%$ & $<0.00001$ & $0.34[-0.12,0.79]$ & 0.15 \\
\hline $\mathrm{FPG}$ & 14 & 1611 & 0.03 & $47 \%$ & 0.03 & $-0.02[-0.16,0.12]$ & 0.80 \\
\hline $\mathrm{HbA} 1 \mathrm{c}$ & 13 & 1543 & 0.02 & $41 \%$ & 0.06 & $0.01[-0.13,0.14]$ & 0.91 \\
\hline PPBS & 11 & 1099 & 0.18 & $81 \%$ & $<0.00001$ & $-0.27[-0.56,0.01]$ & 0.06 \\
\hline $\mathrm{TC}$ & 9 & 887 & 0.16 & $79 \%$ & $<0.0001$ & $0.33[0.03,0.63]$ & 0.03 \\
\hline $\mathrm{HDL}$ & 9 & 887 & 0.24 & $85 \%$ & $<0.00001$ & $0.11[-0.25,0.46]$ & 0.56 \\
\hline LDL & 6 & 702 & 0.02 & $29 \%$ & 0.21 & $0.35[0.16,0.53]$ & 0.0002 \\
\hline $\mathrm{TG}$ & 9 & 887 & 0.05 & $54 \%$ & 0.03 & $0.26[0.05,0.46]$ & 0.01 \\
\hline FINS & 10 & 1019 & 0.41 & $91 \%$ & $<0.00001$ & $0.07[-0.35,0.50]$ & 0.73 \\
\hline
\end{tabular}


Table 3 Cochrane's risk of bias

\begin{tabular}{|c|c|c|c|c|c|c|c|}
\hline Source of bias & $\begin{array}{c}\text { Random } \\
\text { sequence } \\
\text { generation }\end{array}$ & $\begin{array}{c}\text { Allocation } \\
\text { concealment }\end{array}$ & $\begin{array}{c}\text { Blinding of } \\
\text { participants and } \\
\text { personnel }\end{array}$ & $\begin{array}{c}\text { Blinding of } \\
\text { outcome } \\
\text { assessment }\end{array}$ & $\begin{array}{l}\text { Incomplete } \\
\text { outcome data }\end{array}$ & $\begin{array}{l}\text { Selective } \\
\text { reporting }\end{array}$ & $\begin{array}{c}\text { Other source } \\
\text { of bias }\end{array}$ \\
\hline Ling (2003)[33] & $u$ & U & U & U & L & $u$ & $u$ \\
\hline Ramachandran (2004)[34] & $u$ & $U$ & $U$ & U & L & $u$ & $u$ \\
\hline Derosa (2004)[35] & $u$ & $u$ & $U$ & U & $L$ & $u$ & U \\
\hline Gonzolez (2004)[36] & $u$ & L & L & L & L & $U$ & $U$ \\
\hline Rong (2004)[22] & $u$ & $U$ & $U$ & U & L & $U$ & $u$ \\
\hline Tang (2004)[37] & $u$ & $U$ & $U$ & U & $L$ & $u$ & U \\
\hline Yamanouchi (2005)[20] & $\mathrm{L}$ & $\mathrm{L}$ & L & U & $\mathrm{L}$ & $U$ & $u$ \\
\hline Ning (2006)[38] & $U$ & U & $U$ & U & L & $U$ & U \\
\hline Wu (2007)[39] & $u$ & $U$ & $U$ & U & $L$ & $U$ & $u$ \\
\hline Xu (2007)[40] & $U$ & $U$ & $U$ & U & $L$ & $U$ & U \\
\hline Li (2007)[41] & $U$ & $U$ & $U$ & U & L & $U$ & U \\
\hline Wang (2009)[21] & $U$ & $U$ & $U$ & U & $L$ & $U$ & U \\
\hline Rahman (2011)[42] & $U$ & $U$ & $U$ & U & $L$ & $U$ & $u$ \\
\hline Yoon (2011)[21] & $U$ & $U$ & $U$ & U & $\mathrm{L}$ & $U$ & U \\
\hline Wang (2011)[43] & $U$ & $U$ & $U$ & U & L & $U$ & $U$ \\
\hline
\end{tabular}

Note: $L$, low risk of bias; $\mathrm{U}$, unclear risk of bias; $\mathrm{H}$, high risk of bias.

(high-density lipoprotein), LDL (low-density lipoprotein), TG (triglycerides) and FINS (fasting plasma insulin).

\section{Exclusion criteria}

This study excluded the RCTs with the participants who were non-responders to metformin or glimepiride and received dosages exceeded the upper recommended limit (metformin: $2550 \mathrm{mg}$ daily; glimepiride: $8 \mathrm{mg}$ daily) [28]. The RCTs of extremely small sample size (fewer than 10 patients) were also excluded.

\section{Search and selection of studies}

Bibliographical databases, including PubMed, Cochrance Library, Science Direct, China Academic Journals Web Publishing Database, China Master Theses Full-text Database and China Doctor Theses Full-text Database on the China National Knowledge Infrastructure (CNKI), WanFang Data and Google, were searched from their inceptions to 25 Mar 2013.

Search strategies were specified in the working languages of databases, although the terminologies in searching Chinese and English databases were equivalent. Basically, the articles with the terms "glimepiride" and "metformin" in titles, abstracts, and keywords were retriered. Specific search strategies were:

PubMed: "Glimepiride" and "Metformin" in Abstract or Title; Cochrane Llibrary: "Glimepiride" and "Metformin" in Title, Abstract or Keywords; Science Direct: "Glimepiride" and "Metformin" in Abstract, Title, or Keywords; China Academic Jounrnals Web Publishing Database, China Doctor Theses Full-text Database and China Master Theses Full-text Database: 'Title = Glimepiride

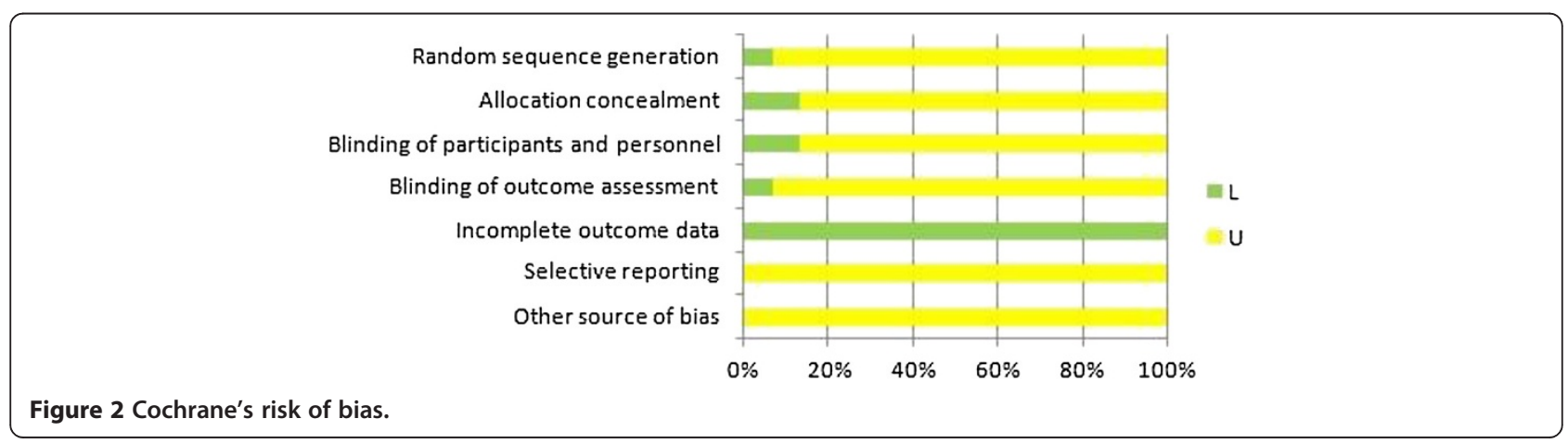


Table 4 Sensitivity analysis (sample size $\geq 90$ )

\begin{tabular}{|c|c|c|c|c|c|c|c|}
\hline \multirow[t]{2}{*}{ Outcome } & \multirow{2}{*}{$\begin{array}{l}\text { No. of } \\
\text { studies }\end{array}$} & \multirow{2}{*}{$\begin{array}{c}\text { Pooled } \\
\text { sample size }\end{array}$} & \multicolumn{3}{|c|}{ Heterogeneity } & \multicolumn{2}{|c|}{ Overall effect } \\
\hline & & & $\mathrm{T}^{2}$ & $1^{2}$ & $P$-value & SMD [95\% Cl] & $\overline{P \text {-value }}$ \\
\hline $\mathrm{BMI}$ & 4 & 605 & 0.05 & $65 \%$ & 0.03 & $0.21[-0.06,0.49]$ & 0.13 \\
\hline FPG & 8 & 1228 & 0.01 & $30 \%$ & 0.18 & $0.09[-0.04,0.23]$ & 0.18 \\
\hline $\mathrm{HbA1c}$ & 8 & 1228 & 0.03 & $56 \%$ & 0.03 & $0.06[-0.12,0.23]$ & 0.52 \\
\hline PPBS & 6 & 792 & 0.00 & $0 \%$ & 0.59 & $-0.01[-0.15,0.13]$ & 0.85 \\
\hline TC & 3 & 504 & 0.22 & $90 \%$ & $<0.0001$ & $0.56[0.00,1.12]$ & 0.05 \\
\hline $\mathrm{HDL}$ & 3 & 504 & 0.18 & $88 \%$ & 0.0002 & $-0.27[-0.78,0.24]$ & 0.30 \\
\hline LDL & 3 & 504 & 0.01 & $21 \%$ & 0.28 & $0.41[0.21,0.61]$ & $<0.0001$ \\
\hline $\mathrm{TG}$ & 3 & 504 & 0.00 & $0 \%$ & 0.61 & $0.13[-0.05,0.30]$ & 0.16 \\
\hline FINS & 5 & 698 & 0.44 & $93 \%$ & $<0.00001$ & $0.37[-0.23,0.98]$ & 0.22 \\
\hline
\end{tabular}

* Metformin or Keyword = Glimepiride * Metformin or Abstract $=$ Glimepiride * Metformin' (in Chinese); Wan Fang: 'Title All "Glimepiride Metformin" or Keywords All "Glimepiride Metformin" or Abstract All "Glimepiride Metformin"' (in Chinese).

Two groups of reviewers (three reviewers in each group) independently performed the literature search and selection. The results from one group were cross- checked by the other group. Disagreements were resolved by group discussion.

\section{Data extraction and quality assessment}

Two reviewers (HZ, XZ) independently extracted data of study characteristics and outcome measures from the selected RCTs. The extracted data were cross-checked before quality assessment according to the Cochrane's

Table 5 Sensitivity analysis based on drug treatment before trials

\begin{tabular}{|c|c|c|c|c|c|c|c|c|}
\hline \multirow[t]{2}{*}{ Criteria } & \multirow[t]{2}{*}{ Outcome } & \multirow{2}{*}{$\begin{array}{l}\text { No. of } \\
\text { studies }\end{array}$} & \multirow{2}{*}{$\begin{array}{c}\text { Pooled } \\
\text { sample size }\end{array}$} & \multicolumn{3}{|c|}{ Heterogeneity } & \multicolumn{2}{|c|}{ Overall effect } \\
\hline & & & & $\mathrm{T}^{2}$ & $1^{2}$ & P-value & SMD [95\% CI] & P-value \\
\hline \multirow{11}{*}{$\begin{array}{l}\text { Excluding the studies } \\
\text { with non-responders to } \\
\text { other sulfonylureas }\end{array}$} & BMI & 10 & 988 & 0.50 & $92 \%$ & $<0.00001$ & $-0.06[-0.53,0.40]$ & 0.79 \\
\hline & SBP & 5 & 615 & 0.22 & $86 \%$ & $<0.0001$ & $0.39[-0.06,0.83]$ & 0.09 \\
\hline & DBP & 5 & 615 & 0.23 & $87 \%$ & $<0.00001$ & $0.34[-0.12,0.79]$ & 0.15 \\
\hline & FPG & 12 & 1314 & 0.04 & $48 \%$ & 0.03 & $0.01[-0.15,0.17]$ & 0.91 \\
\hline & $\mathrm{HbA} 1 \mathrm{c}$ & 11 & 1246 & 0.03 & $46 \%$ & 0.05 & $0.04[-0.12,0.19]$ & 0.65 \\
\hline & PPBS & 9 & 802 & 0.27 & $85 \%$ & $<0.00001$ & $-0.32[-0.69,0.06]$ & 0.10 \\
\hline & $\mathrm{TC}$ & 9 & 887 & 0.16 & $79 \%$ & $<0.0001$ & $0.33[0.03,0.63]$ & 0.03 \\
\hline & $\mathrm{HDL}$ & 9 & 887 & 0.24 & $85 \%$ & $<0.00001$ & $0.11[-0.25,0.46]$ & 0.56 \\
\hline & LDL & 6 & 702 & 0.02 & $29 \%$ & 0.21 & $0.35[0.16,0.53]$ & 0.0002 \\
\hline & TG & 9 & 887 & 0.05 & $54 \%$ & 0.03 & $0.26[0.05,0.46]$ & 0.01 \\
\hline & FINS & 8 & 722 & 0.58 & $92 \%$ & $<0.00001$ & $0.11[-0.44,0.67]$ & 0.69 \\
\hline \multirow{11}{*}{$\begin{array}{l}\text { Excluding the studies with } \\
\text { participants who used other } \\
\text { anti-diabetic drugs before RCTs }\end{array}$} & BMI & 6 & 687 & 0.05 & $56 \%$ & 0.04 & $0.27[0.03,0.51]$ & 0.03 \\
\hline & SBP & 3 & 444 & 0.22 & $87 \%$ & 0.0004 & $0.60[0.03,1.16]$ & 0.04 \\
\hline & DBP & 3 & 444 & 0.29 & $90 \%$ & $<0.0001$ & $0.52[-0.12,1.16]$ & 0.11 \\
\hline & FPG & 8 & 989 & 0.07 & $66 \%$ & 0.004 & $-0.03[-0.26,0.19]$ & 0.78 \\
\hline & $\mathrm{HbA} 1 \mathrm{c}$ & 7 & 921 & 0.05 & $61 \%$ & 0.02 & $-0.00[-0.22,0.22]$ & 1.00 \\
\hline & PPBS & 5 & 477 & 0.54 & $92 \%$ & $<0.00001$ & $-0.60[-1.28,0.08]$ & 0.08 \\
\hline & $\mathrm{TC}$ & 6 & 687 & 0.18 & $82 \%$ & $<0.0001$ & $0.38[0.00,0.76]$ & 0.05 \\
\hline & $\mathrm{HDL}$ & 6 & 687 & 0.12 & $76 \%$ & 0.0009 & $-0.14[-0.46,0.19]$ & 0.41 \\
\hline & LDL & 4 & 572 & 0.04 & $56 \%$ & 0.08 & $0.30[0.05,0.56]$ & 0.02 \\
\hline & TG & 6 & 687 & 0.00 & $0 \%$ & 0.74 & $0.13[-0.02,0.28]$ & 0.10 \\
\hline & FINS & 5 & 483 & 0.53 & $92 \%$ & $<0.00001$ & $0.43[-0.24,1.10]$ & 0.21 \\
\hline
\end{tabular}


risks of bias tool [29]. Disagreement was resolved by discussion between the reviewers (HZ, XZ). A third reviewer (SZ) was consulted when necessary.

\section{Meta-analysis}

Extracted data were transferred to Review Manager 5.2 [30] for meta-analysis with random-effects model. Numeric outcome measures were represented in standardized mean differences (SMD) or odds ratios (OR) and their $95 \%$ confidence intervals (CI). Study heterogeneity was evaluated with $\mathrm{T}^{2}$ test and $\mathrm{I}^{2}$ statistics. $\mathrm{P}$ values below 0.05 were considered statistically significant.

\section{Sensitivity and subgroup analysis}

Sensitivity analysis of the efficacy was performed on the sample sizes, on whether the patients received prior antidiabetic treatments and on the daily dose of metformin. Subgroup analysis was performed to explain the heterogeneity in terms of differences in follow-up periods and characteristics of the participants.

Table 6 Sensitivity analysis based on the daily doses of metformin

\begin{tabular}{|c|c|c|c|c|c|c|c|c|}
\hline \multirow[t]{2}{*}{ Daily doses } & \multirow[t]{2}{*}{ Outcome } & \multirow{2}{*}{$\begin{array}{l}\text { No. of } \\
\text { studies }\end{array}$} & \multirow{2}{*}{$\begin{array}{c}\text { Pooled } \\
\text { sample size }\end{array}$} & \multicolumn{3}{|c|}{ Heterogeneity } & \multicolumn{2}{|c|}{ Overall effect } \\
\hline & & & & $\mathrm{T}^{2}$ & $1^{2}$ & $P$ P-value & SMD [95\% Cl] & $\overline{P \text {-value }}$ \\
\hline \multirow[t]{11}{*}{ Below 1000 mg only } & BMI & 4 & 352 & 0.01 & $22 \%$ & 0.28 & $0.33[0.09,0.58]$ & 0.007 \\
\hline & SBP & 2 & 177 & 0.00 & $0 \%$ & 0.64 & $-0.02[-0.31,0.28]$ & 0.92 \\
\hline & DBP & 2 & 177 & 0.00 & $0 \%$ & 0.67 & $-0.06[-0.36,0.23]$ & 0.69 \\
\hline & FPG & 5 & 584 & 0.02 & $34 \%$ & 0.19 & $0.03[-0.18,0.24]$ & 0.77 \\
\hline & $\mathrm{HbA1c}$ & 5 & 584 & 0.07 & $65 \%$ & 0.02 & $0.14[-0.15,0.44]$ & 0.34 \\
\hline & PPBS & 3 & 276 & 0.04 & $46 \%$ & 0.16 & $0.02[-0.32,0.36]$ & 0.91 \\
\hline & TC & 3 & 251 & 0.04 & $41 \%$ & 0.18 & $0.32[-0.03,0.66]$ & 0.07 \\
\hline & $\mathrm{HDL}$ & 3 & 251 & 0.00 & $0 \%$ & 0.83 & $0.01[-0.23,0.26]$ & 0.91 \\
\hline & LDL & 1 & 136 & NA & NA & NA & $0.19[-0.15,0.53]$ & 0.27 \\
\hline & $\mathrm{TG}$ & 3 & 251 & 0.00 & $0 \%$ & 0.67 & $0.10[-0.15,0.34]$ & 0.44 \\
\hline & FINS & 4 & 352 & 0.00 & $4 \%$ & 0.37 & $0.22[0.00,0.43]$ & 0.05 \\
\hline \multirow[t]{11}{*}{$1000 \mathrm{mg}$ and above $1000 \mathrm{mg}$} & BMI & 6 & 636 & 0.87 & $95 \%$ & $<0.00001$ & $-0.36[-1.13,0.41]$ & 0.36 \\
\hline & SBP & 3 & 438 & 0.17 & $85 \%$ & 0.002 & $0.64[0.12,1.16]$ & 0.02 \\
\hline & DBP & 3 & 438 & 0.19 & $86 \%$ & 0.0009 & $0.60[0.06,1.14]$ & 0.03 \\
\hline & FPG & 9 & 1027 & 0.04 & $55 \%$ & 0.02 & $-0.05[-0.24,0.14]$ & 0.64 \\
\hline & $\mathrm{HbA1c}$ & 8 & 959 & 0.00 & $0 \%$ & 0.87 & $-0.08[-0.21,0.04]$ & 0.20 \\
\hline & PPBS & 8 & 823 & 0.22 & $84 \%$ & $<0.00001$ & $-0.37[-0.73,-0.01]$ & 0.04 \\
\hline & TC & 6 & 636 & 0.24 & $85 \%$ & $<0.00001$ & $0.34[-0.09,0.77]$ & 0.12 \\
\hline & $\mathrm{HDL}$ & 6 & 636 & 0.39 & $90 \%$ & $<0.00001$ & $0.14[-0.39,0.68]$ & 0.59 \\
\hline & LDL & 5 & 566 & 0.02 & $32 \%$ & 0.21 & $0.38[0.17,0.59]$ & 0.0004 \\
\hline & $\mathrm{TG}$ & 6 & 636 & .0 .08 & $67 \%$ & 0.010 & $0.36[0.07,0.64]$ & 0.02 \\
\hline & FINS & 6 & 667 & 0.71 & $95 \%$ & $<0.00001$ & $-0.02[-0.72,0.67]$ & 0.94 \\
\hline \multirow[t]{11}{*}{ Above 1000 mg only } & BMI & 2 & 234 & 0.11 & $72 \%$ & 0.06 & $0.16[-0.38,0.69]$ & 0.56 \\
\hline & SBP & 2 & 234 & 0.42 & $91 \%$ & 0.001 & $0.69[-0.25,1.63]$ & 0.15 \\
\hline & DBP & 2 & 234 & 0.47 & $92 \%$ & 0.0005 & $0.63[-0.36,1.63]$ & 0.21 \\
\hline & FPG & 3 & 461 & 0.07 & $73 \%$ & 0.02 & $0.02[-0.35,0.38]$ & 0.93 \\
\hline & $\mathrm{HbA1c}$ & 3 & 461 & 0.00 & $0 \%$ & 0.91 & $-0.16[-0.34,0.03]$ & 0.10 \\
\hline & PPBS & 3 & 461 & 0.00 & $0 \%$ & 0.82 & $-0.11[-0.29,0.07]$ & 0.24 \\
\hline & $\mathrm{TC}$ & 2 & 234 & 0.01 & $17 \%$ & 0.27 & $0.23[-0.07,0.52]$ & 0.13 \\
\hline & $\mathrm{HDL}$ & 2 & 234 & 0.00 & $0 \%$ & 0.48 & $0.06[-0.20,0.32]$ & 0.64 \\
\hline & LDL & 1 & 164 & NA & NA & NA & $0.43[0.12,0.74]$ & 0.006 \\
\hline & TG & 2 & 234 & 0.04 & $49 \%$ & 0.16 & $0.16[-0.23,0.55]$ & 0.42 \\
\hline & FINS & 4 & 531 & 0.71 & $95 \%$ & $<0.00001$ & $0.28[-0.57,1.13]$ & 0.52 \\
\hline
\end{tabular}




\section{Adverse events analysis}

Adverse events analysis was performed on the hypoglycemia, gastrointestinal upset and overall side effects with their odds ratios (OR) and 95\% CI.

\section{Publication bias}

Funnel plots were generated to visualize possible publication bias. Begg and Egger's tests using the package "metafor" [31] with statistical software R [32] evaluated the statistical significance of the publication bias.

\section{Results}

\section{Included studies and their characteristics}

Figure 1 shows the selection process of the studies. A total of 1023 records were identified in accordance with the search strategies from specific bibliographical databases, i.e. PubMed $(n=208)$, Cochrance Library $(n=89)$, Science Direct $(n=48)$, Chinese National Knowledge Infrastructure $(n=267)$, WangFang $(n=206)$ and Google $(n=205)$. Among the 440 records after removal of duplicates, 27 records met the eligibility criteria. After full-text assessment, 12 of 27 studies were excluded for the reasons stated in Figure 1. As a result, 15 RCTs with 1681 participants were included for meta-analysis. The characteristics of the included studies are shown in Table 1.

\section{Overall effects}

Meta-analysis was performed on the outcome measures FPG, BMI, HbA1c, PPBS, TC, FINS, HDL, LDL, TG, SBP and DBP. The SMD, 95\% CI and P values for outcomes between metformin and glimepiride are shown in Table 2. The SMDs between metformin and glimepiride were only statistically significant on TC $(0.33[0.03$, 0.63 ], $\mathrm{P}=0.03)$, LDL $(0.35$ [0.16, 0.53], $\mathrm{P}=0.00002)$, and TG $(0.26[0.05,0.46], P=0.01)$, indicating that efficacy of metformin was statistically significant over glimepiride in lipid metabolism indices. The differences in glycemic control (e.g. HbA1c and PPBS) and cardiovascular indices (e.g. blood pressure) were not statistically significant. As shown in Table 2, there were significant heterogeneities among studies in SBP $\left(\mathrm{I}^{2}=86 \%, \mathrm{P}<0.0001\right)$, DBP $\left(\mathrm{I}^{2}=87 \%, \mathrm{P}<0.00001\right)$, PPBS $\left(\mathrm{I}^{2}=81 \%, \mathrm{P}<0.00001\right), \mathrm{TC}$ $\left(\mathrm{I}^{2}=79 \%, \mathrm{P}<0.0001\right), \mathrm{HDL}\left(\mathrm{I}^{2}=86 \%, \mathrm{P}<0.00001\right)$ and FINS $\quad\left(\mathrm{I}^{2}=91 \%, \quad \mathrm{P}<0.00001\right) . \quad$ The heterogeneities justified the use of random-effects model in meta-analysis.

\section{Risk of bias across studies}

Cochrane's risk of bias tool was used to assess the RCT quality (Table 3 and Figure 2). The attrition bias of all included studies was low (few missing data). Other key aspects among studies were mostly unclear in risk of bias except two studies $[20,36]$.

Table 7 Subgroup analysis of different follow-up periods

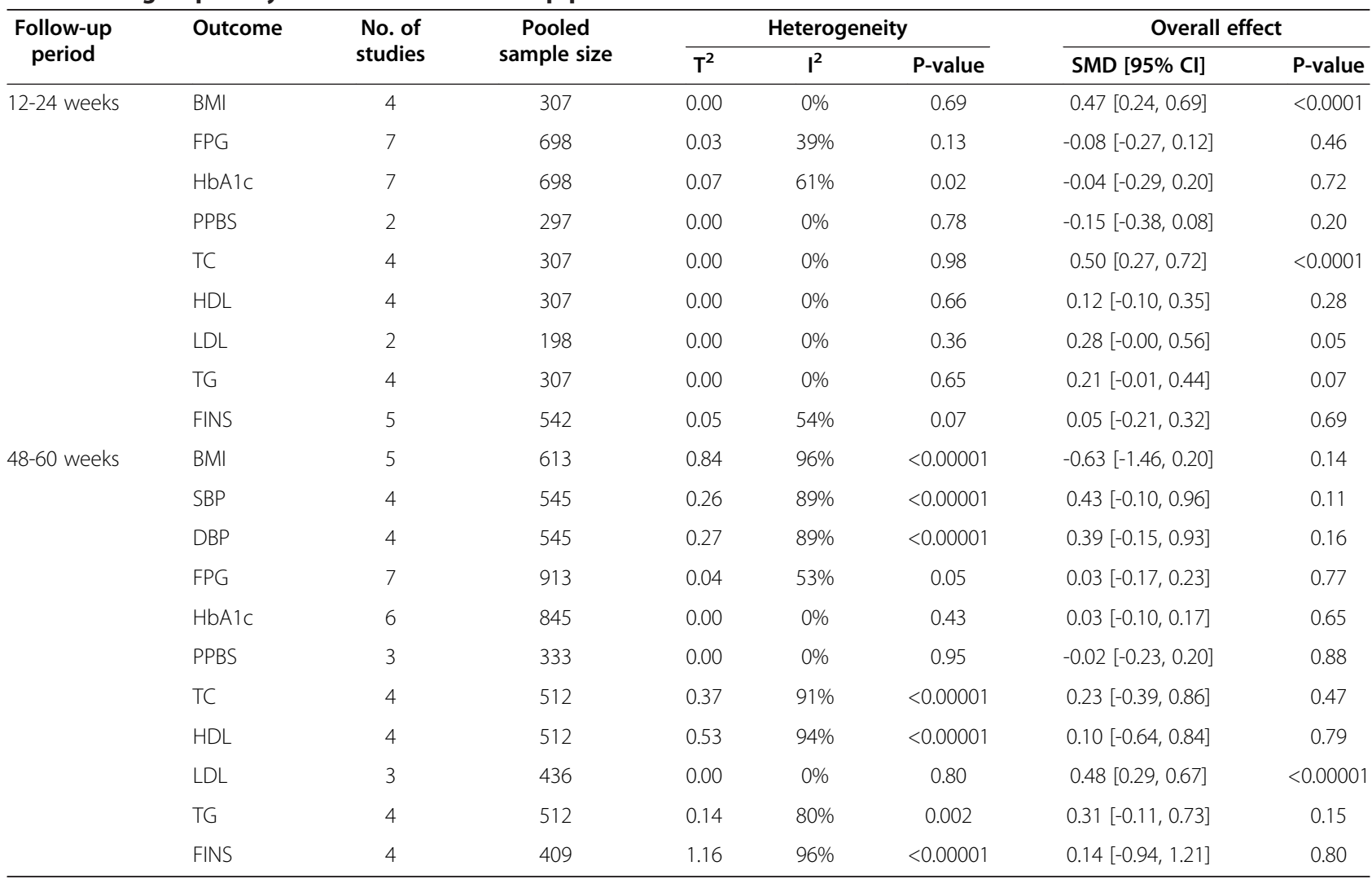




\section{Sensitivity analysis}

Sensitivity analysis checked whether the overall effects would be different if only the studies with the sample size $\mathrm{N} \geq 90$ were included. As shown in Table 4, metformin outperformed glimepiride only on LDL (0.41 [0.21, 0.61 ], $\mathrm{P}<0.0001$ ) in the studies with sample size $\mathrm{N} \geq 90$. Other outcomes such as FPG, BMI, TC and TG did not show significant difference between glimepiride and metformin.

Sensitivity analysis also considered whether prior use of anti-diabetic drugs would affect the RCT results. Table 5 shows that only the effect of metformin on BMI became statistically significant after excluding the studies with participants who were non-responders to other sulfonyureas. The significance of the effects of metformin on TC, LDL, TG and other aspects remained the same, indicating the overall results on those aspects were robust.

Sensitivity analysis tested whether the efficiency of metformin would be different if the daily dose of metformin was less than $1000 \mathrm{mg}$. Table 6 shows that metformin outperformed glimepiride only on BMI (0.33 [0.09,
0.58], $\mathrm{P}=0.007)$ with a low daily dose and on LDL $(0.43$ $[0.12,0.74], \mathrm{P}=0.006)$ with a relative high daily dose.

\section{Subgroup analysis}

Meta-analysis of the subgroups with different follow-up periods (12-24 weeks and 48-60 weeks) showed that metformin moderated BMI and TC better than glimepiride in the shorter term while both drugs were equivalent in performance in all aspects except LDL in the longer term. As shown in Table 7, metformin performed better than glimepiride on both BMI $(0.47$ [0.24, 0.69], $\mathrm{P}<$ $0.0001)$ and TC $(0.50[0.27,0.72], \mathrm{P}<0.0001)$ in $12-24$ weeks subgroup. In 48-60 weeks subgroup, metformin performed better only on LDL $(0.48$ [0.29, 0.67], P < 0.00001).

Meta-analysis of the subgroups with BMI below or above 27.5 (i.e. the norm in the countries where the included RCTs were conducted) [44] was also conducted. As shown in Table 8, metformin outperformed glimepiride on control of FPG (0.34 [0.11, 0.57], $\mathrm{P}=0.003)$, TC (0.33 [0.08, 0.58], $\mathrm{P}=0.01)$ and $\operatorname{LDL}(0.32[0.08,0.56]$, $\mathrm{P}=0.008)$ in the higher $\mathrm{BMI}$ subgroup (BMI $\geq 27.5)$. However, in the lower BMI subgroup $(\mathrm{BMI}<27.5)$

Table 8 Subgroup analysis of different BMI

\begin{tabular}{|c|c|c|c|c|c|c|c|c|}
\hline \multirow[t]{2}{*}{ BMI } & \multirow[t]{2}{*}{ Outcome } & \multirow{2}{*}{$\begin{array}{l}\text { No. of } \\
\text { studies }\end{array}$} & \multirow{2}{*}{$\begin{array}{c}\text { Pooled } \\
\text { sample size }\end{array}$} & \multicolumn{3}{|c|}{ Heterogeneity } & \multicolumn{2}{|c|}{ Overall effect } \\
\hline & & & & $\mathrm{T}^{2}$ & $\mathrm{I}^{2}$ & P-value & SMD [95\% Cl] & P-value \\
\hline \multirow[t]{11}{*}{$\mathrm{BMI}<27$} & $\mathrm{BMI}$ & 7 & 618 & 0.83 & $94 \%$ & $<0.00001$ & $-0.27[-0.98,0.43]$ & 0.45 \\
\hline & SBP & 3 & 381 & 0.09 & $72 \%$ & 0.03 & $0.20[-0.21,0.60]$ & 0.34 \\
\hline & $\mathrm{DBP}$ & 3 & 381 & 0.10 & $74 \%$ & 0.02 & $0.15[-0.27,0.57]$ & 0.48 \\
\hline & $\mathrm{FPG}$ & 7 & 788 & 0.02 & $38 \%$ & 0.14 & $-0.05[-0.24,0.13]$ & 0.57 \\
\hline & $\mathrm{HbA1c}$ & 6 & 720 & 0.00 & $0 \%$ & 0.43 & $0.06[-0.09,0.20]$ & 0.43 \\
\hline & PPBS & 4 & 276 & 0.23 & $780 \%$ & 0.003 & $-0.38[-0.91,0.15]$ & 0.16 \\
\hline & $\mathrm{TC}$ & 5 & 455 & 0.37 & $88 \%$ & $<0.00001$ & $0.26[-0.31,0.84]$ & 0.37 \\
\hline & HDL & 5 & 455 & 0.57 & $92 \%$ & $<0.00001$ & $0.11[-0.58,0.81]$ & 0.75 \\
\hline & LDL & 3 & 340 & 0.08 & $65 \%$ & 0.06 & $0.30[-0.09,0.70]$ & 0.14 \\
\hline & $\mathrm{TG}$ & 5 & 455 & 0.13 & $72 \%$ & 0.006 & $0.33[-0.05,0.71]$ & 0.09 \\
\hline & FINS & 5 & 352 & 0.21 & $78 \%$ & 0.001 & $-0.17[-0.63,0.29]$ & 0.47 \\
\hline \multirow[t]{11}{*}{$\mathrm{BMI} \geq 27$} & $\mathrm{BMI}$ & 3 & 370 & 0.13 & $79 \%$ & 0.009 & $0.31[-0.15,0.78]$ & 0.18 \\
\hline & SBP & 2 & 234 & 0.42 & $91 \%$ & 0.001 & $0.69[-0.25,1.63]$ & 0.15 \\
\hline & DBP & 2 & 234 & 0.47 & $92 \%$ & 0.0005 & $0.63[-0.36,1.63]$ & 0.21 \\
\hline & FPG & 2 & 300 & 0.00 & $0 \%$ & 0.82 & $0.34[0.11,0.57]$ & 0.003 \\
\hline & $\mathrm{HbA} 1 \mathrm{c}$ & 2 & 300 & 0.27 & $91 \%$ & 0.001 & $0.17[-0.58,0.93]$ & 0.65 \\
\hline & PPBS & 2 & 300 & 0.01 & $29 \%$ & 0.24 & $0.09[-0.18,0.36]$ & 0.51 \\
\hline & $\mathrm{TC}$ & 3 & 370 & 0.02 & $30 \%$ & 0.24 & $0.33[0.08,0.58]$ & 0.01 \\
\hline & $\mathrm{HDL}$ & 3 & 370 & 0.00 & $0 \%$ & 0.71 & $0.03[-0.18,0.23]$ & 0.79 \\
\hline & LDL & 2 & 300 & 0.00 & $6 \%$ & 0.30 & $0.32[0.08,0.56]$ & 0.008 \\
\hline & $\mathrm{TG}$ & 3 & 370 & 0.00 & $4 \%$ & 0.35 & $0.15[-0.06,0.36]$ & 0.17 \\
\hline & FINS & 3 & 370 & 0.76 & $95 \%$ & $<0.00001$ & $0.56[-0.45,1.57]$ & 0.28 \\
\hline
\end{tabular}


Table 9 Adverse events

\begin{tabular}{|c|c|c|c|c|c|c|c|}
\hline \multirow[t]{2}{*}{ Adverse events } & \multirow{2}{*}{$\begin{array}{l}\text { No. of } \\
\text { studies }\end{array}$} & \multirow{2}{*}{$\begin{array}{c}\text { Pooled } \\
\text { sample size }\end{array}$} & \multicolumn{3}{|c|}{ Heterogeneity } & \multicolumn{2}{|c|}{ Overall effect } \\
\hline & & & $\overline{\mathrm{T}^{2}}$ & $1^{2}$ & P-value & OR $[95 \% \mathrm{Cl}]$ & P-value \\
\hline All side effects & 8 & 1003 & 4.70 & $81 \%$ & $<0.00001$ & $0.35[0.06,2.01]$ & 0.24 \\
\hline Hypoglycemia & 5 & 542 & 0.00 & $0 \%$ & 0.77 & $4.94[2.03,11.99]$ & 0.0004 \\
\hline Gastrointestinal upset & 5 & 763 & 2.27 & $61 \%$ & 0.04 & $0.07[0.01,0.37]$ & 0.002 \\
\hline
\end{tabular}

metformin and glimepiride were not significantly different in performance as determined by outcome measures.

\section{Adverse events}

Eight out of 15 studies reported adverse events. As shown in Table 9, glimepiride had more hypoglycemia episodes than metformin did $(4.94[2.03,11.99], \mathrm{P}=0.0004)$. Incidents of gastrointestinal upset, including diarrhea, epigastric discomfort, stomach pain and abdominal distension, were reported more frequently in metformin group ( 0.07 $[0.01,0.37], \mathrm{P}=0.002)$. Overall, these two drugs had no significant difference in side effects $(0.35$ [0.06, 2.01], $\mathrm{P}=0.24)$ among the included RCTs.

\section{Publication bias}

Funnel plots were generated to visualize possible publication bias. Major outcomes FPG, BMI, HbA1c, PPBS, TC, FINS, HDL, LDL, TG, SBP and DBP showed moderate asymmetries across studies in the funnel plots, indicating there was publication bias. A typical funnel plot is shown in Figure 3. The statistical significance of the moderate publication bias in sugar (FPG and PPBS) and lipid (HDL and TG) indices was confirmed by the Begg's rank correlation test [45]. Egger's linear regression method [46] further confirmed the statistical significance of the publication bias in FPG towards metformin (Table 10), indicating that the difference in glycemic control efficacy between metformin and glimepiride was less than it seemed.

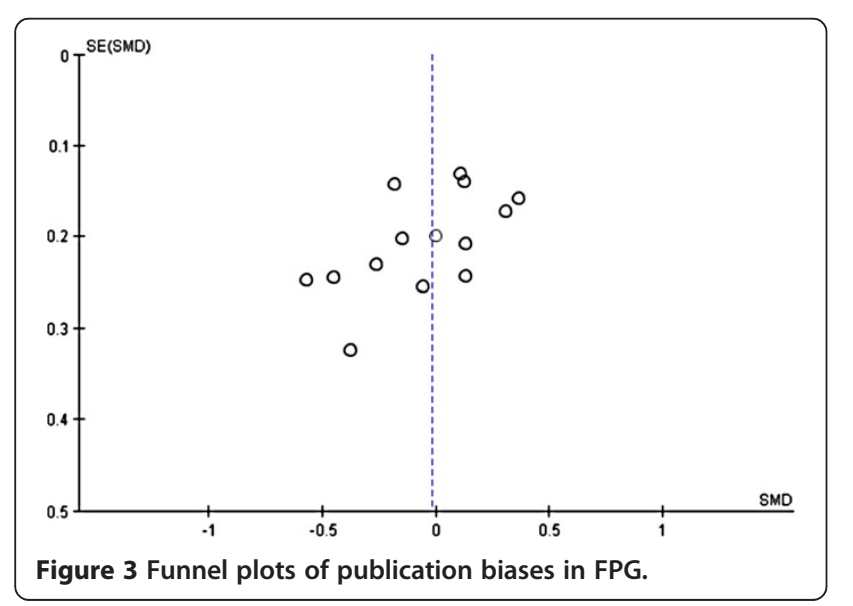

\section{Discussion}

Metformin remains the most effective monotherapy of T2DM while its advantages are diminishing among newer anti-diabetic drugs. Past studies comparing the efficacy between metformin with sulfonylureas showed that metformin was significantly better in controlling HbA1c, FPG, BMI, LDL and TG [3-5,7]. Glimepiride is a better sulphonylurea in treating T2DM $[17-19,47,48]$. The present meta-analytic study found that metformin was not significantly better than glimepiride, particularly in controlling HbA1c, FPG and BMI.

This meta-analysis supported that both metformin and glimepiride was effective in treating T2DM for glycemic control. Metformin performed better than glimepiride in management of BMI and lipid metabolism indices but the advantages of metformin were only significant in short follow-up periods.

These results were deemed robust after evaluation by sensitivity analysis that excluded small RCTs and the participants who were non-responders to non-glimepiride sulfonylureas or who received anti-diabetic treatment. The differences between metformin and glimepiride became insignificant in large RCTs. Even for treating the patients who were not responding to conventional (non-glimepiride) sulfonylureas, glimepiride and metformin were equivalent in glycemic control. This finding could not be achieved by comparing sulfonylureas (including glimepiride) as a group with metformin.

Table 10 Results of Begg and Egger's tests

\begin{tabular}{|c|c|c|c|c|c|}
\hline \multirow[t]{2}{*}{ Outcome } & \multirow{2}{*}{$\begin{array}{l}\text { No. of } \\
\text { studies }\end{array}$} & \multicolumn{2}{|c|}{ Begg's test } & \multicolumn{2}{|c|}{ Egger's test } \\
\hline & & $z$ & P-value & Kendall's tau & P-value \\
\hline $\mathrm{BMI}$ & 10 & -2.9174 & 0.0035 & -0.0222 & 1.0000 \\
\hline SBP & 5 & -1.3229 & 0.1859 & -0.2000 & 0.1867 \\
\hline DBP & 5 & -1.5941 & 0.1109 & -0.4000 & 0.4833 \\
\hline $\mathrm{FPG}$ & 14 & -2.2496 & 0.0245 & -0.4286 & 0.0356 \\
\hline $\mathrm{HbAlc}$ & 13 & -0.9014 & 0.3673 & -0.2308 & 0.3062 \\
\hline PPBS & 11 & -2.2267 & 0.0260 & -0.4182 & 0.0866 \\
\hline $\mathrm{TC}$ & 9 & -0.8218 & 0.4112 & -0.0556 & 0.9195 \\
\hline $\mathrm{HDL}$ & 9 & 2.2070 & 0.0273 & 0.5000 & 0.0752 \\
\hline LDL & 6 & -1.1076 & 0.2680 & -0.3333 & 0.4694 \\
\hline $\mathrm{TG}$ & 9 & 0.8232 & 0.04104 & 0.2778 & 0.3585 \\
\hline FINS & 10 & -0.8661 & 0.3864 & -0.2444 & 0.3807 \\
\hline
\end{tabular}


Adverse events analysis showed that glimepiride had more hypoglycemia episodes, in agreement with previous results that metformin was associated with less hypoglycemia than sulphonylureas [3] and that metformin had a higher risk of gastrointestinal upset [49-51]. The weight gain side effects were not significant in the included RCTs, in consistency with the findings of previous studies [52] on the weight gain issue of glimepiride.

The daily dose of metformin affected the efficacy and side effects of metformin. When the daily dose of metformin was more than $1000 \mathrm{mg}$, the probability of gastrointestinal upset would be increased exponentially [53]. Sensitivity analysis on the daily doses of metformin showed that metformin outperformed glimepiride only on BMI with a low daily dose and on LDL with a relatively high daily dose, but was not significantly better than glimepiride on controlling HbA1c, FPG and Fins in all daily doses.

Most of the included RCTs (13 out of 15 in this metaanalysis) were conducted in Asia; thus, the Asian norm of BMI for subgroup analysis was adopted. It seems that Asian patients were less affected by the weight gain side effect of glimepiride. Multi-country and multi-ethnic trials are warranted to test whether glimepiride is more suitable for Asian patients. Double-blind RCTs with longer follow-up periods should be conducted to assess other side effects such as those on cardiovascular system. It is encouraging that new clinical trials comparing metformin and glimepiride for monotherapy of T2DM have been registered [54]. Hence, proper updates on this meta-analysis will be conducted in forthcoming years.

These results provide direct evidence to support ADA's and NICE's recommendations to consider glimepiride as one of the alternatives to metformin. Our meta-analysis of the RCT results demonstrated that the advantages of metformin over glimepiride were not always significant particularly in Asian patients.

\section{Conclusion}

Metformin and glimepiride were not significantly different in glycemic control of T2DM, suggesting that glimepiride would be a good choice second to metformin in the monotherapy of T2DM.

\footnotetext{
Abbreviations

BMI: Body mass index; DBP: Diastolic blood pressure; FPG: Fasting plasma glucose; FINS: Fasting plasma insulin; HbA1c: Glycosylated hemoglobin level; HDL: High-density lipoprotein; LDL: Low-density lipoprotein; PPBS: Postprandial blood sugar; RCT: Random control trials; SBP: Systolic blood pressure; TC: Total cholesterol; TG: Triglycerides; T2DM: Type 2 diabetes mellitus.
}

\section{Competing interests}

The authors declare that they have no competing interests.

\section{Authors' contributions}

SL supervised this study. $\mathrm{HZ}$ and $\mathrm{XZ}$ extracted and analyzed the data from the selected studies according to the eligibility criteria. SZ repeated data analysis for cross-checking. Other authors assisted in searching the databases, assessing and selecting studies. HZ, SZ and SL interpreted the data and drafted a report on the findings. SL revised the manuscript for submission. All authors read and approved the final version of the manuscript.

\section{Acknowledgement}

The work of HZ, SZ and SL is part of a research project "Open systematic reviewing of clinical trials" (MYRG190-Y3-L3-ICMS-LSW) funded by the University of Macau.

Received: 13 May 2013 Accepted: 4 November 2013

Published: 14 November 2013

\section{References}

1. The National Collaborating Centre for Chronic Conditions: Type 2 diabetes: national clinical guideline for management in primary and secondary care (update). London: Royal College of Physicians; 2008.

2. Association American Diabetes: Standards of medical care in diabetes-2013. Diabetes Care 2013, 36:S11-66.

3. UK Prospective Diabetes Study Group: Effect of intensive blood-glucose control with metformin on complications in overweight patients with type 2 diabetes (UKPDS 34). Lancet 1998, 352:854-865.

4. Stratton IM, Adler Al, Neil HAW, Matthews DR, Manley SE, Cull CA, Hadden D, Turner RC, Holman RR, on behalf of the UK Prosepective Diabetes Study Group: Association of glycaemia with macrovascular and microvascular complications of type 2 diabetes (UKPDS 35): prospective observational study. BMJ 2000, 321:405-412.

5. Adler Al, Stratton IM, Haw N, Yudkin JS, Matthews DR, Cull CA, Wright AD, Turner RC, Holman RR, UK Prospective Diabetes Study Group: Association of systolic blood pressure with macrovascular and microvascular complications of type 2 diabetes (UKPDS 36): prospective observational study. BMJ 2000, 321:412-419.

6. Inzucchi SE, Bergenstal RM, Buse JB, Diamant M, Ferrannini E, Nauck M, Peters AL, Tsapas A, Wender R, Matthews DR: Management of hyperglycemiain type 2 diabetes: a patient-centered approach. Diabetes Care 2012, 35:1364-1379.

7. Saenz A, Fernandez-Esteban I, Mataix A, Ausejo Segura M, Roqué i Figuls M Mohr D: Metformin monotherapy for type 2 diabetes mellitus. Cochrane DB Syst Rev 2009, 3:CD002966. doi: 10.1002/14651858.CD002966.pub3.

8. Holman RR, Paul SK, Bethel MA, Matthews DR, Neil HAW: 10-year follow-up of intensive glucose control in type 2 diabetes. NEJM 2008, 359:1577-1589.

9. Bennett WL, Maruthur NM, Singh S, Segal JB, Wilson LM, Chatterjee R, Marinopoulos SS, Puhan MA, Ranasinghe P, Block L, Nicholson WK, Hutfless S, Bass EB, Bolen S: Comparative effectiveness and safety of medications for type 2 diabetes: an update including new drugs and 2-drug combinations. Ann Intern Med 2011, 154:602-614.

10. Schernthaner $G$, Matthews DR, Charbonnel B, Hanefeld M, Brunetti $P$, on behalf of the quarter study group: Efficacy and safety of pioglitazone versus metformin in patients with type 2 diabetes mellitus: a doubleblind, randomized trial. J Clin Endocrinol Metab 2004, 89:6068-6076.

11. Marre M, Gaal LV, Usadel KH, Ball M, Whatmough I, Guitard C: Nateglinide improves glycaemic control when added to metformin monotherapy: results of a randomized trial with type 2 diabetes patients. Diabetes Obes Metab 2002, 4:177-186.

12. Bailey CJ, Bagdonas A, Rubes J, McMorn SO, Donaldson J, Biswas N, Stewart MW: Rosiglitazone/metformin fixed-dose combination compared with uptitrated metformin alone in type 2 diabetes mellitus: a 24-week, multicenter, randomized, double-blind, parallel-group study. Clin Ther 2005, 27:1548-1561.

13. Cryer DR, Nicholas SP, Henry DH, Mills DJ, Stadel BV: Comparative outcomes study of metformin intervention versus conventional approach. Diabetes Care 2005, 28:539-543.

14. Boussageon R, Suupper I, Bejan-Angoulvant T, Kellou N, Cucherat M, Boissel JP, Kassai B, Moreau A, Gueyffier F, Cornu C: Reappraisal of metformin efficacy in the treatment of type 2 diabetes: a meta-analysis of randomised controlled trials. PLOS Med 2012, 9:e1001204.

15. Rojas LBA, Gomes MB: Metformin: an old but still the best treatment for type 2 diabetes. Diabetol Metab Syndr 2013, 5:6-20. 
16. Hamaguchi T, Hirose T, Asakawa H, Itoh Y, Kamado K, Tokunaga K, Tomita K, Masuda H, Watanabe N, Namba M: Efficacy of glimepiride in type 2 diabetic patients treated with glibenclamide. Diabetes Res Clin Pract 2004, 66:S129-132.

17. Rendell M: The role of sulphonylureas in the management of type 2 diabetes mellitus. Drugs 2004, 64:1339-1358.

18. Nissen SE, Nicholls SJ, Wolski K, Nesto R, Kupfer S, Perez A, Jure H, Larochellière R, Staniloae CS, Mavromatis K, Saw J, Hu B, Lincoff AM, Tuzcu EM, for the PERISCOPE Investigators: Comparison of pioglitazone vs glimepiride on progression of coronary atherosclerosis in patients with type 2 diabetes. JAMA 2008, 299:1561-1573

19. Schotborgh CE, Wilde AAM: Sulfonylurea derivatives in cardiovascular research and in cardiovascular patients. Cardiovasc Res 1997, 34:73-80.

20. Yamanouchi $T$, Sakai $T$, Igarashi $K$, Ichiyanagi $K$, Watanabe $H$, Kawasaki T: Comparison of metabolic effects of pioglitazone, metformin, and glimepiride over 1 year in Japanese patients with newly diagnosed Type 2 diabetes. Diabet Med 2005, 22:980-985.

21. Yoon KH, Shin JA, Kwon HS, Lee SH, Min KW, Ahn YB, Yoo SJ, Ahn KJ, Park SW, Lee KW, Sung YA, Park TS, Kim MS, Kim YK, Nam MS, Kim HS, Park IB, Park JS, Woo JT, Son HY: Comparison of the efficacy of glimepiride, metformin, and rosiglitazone monotherapy in korean drug-naive type 2 diabetic patients: the practical evidence of antidiabetic monotherapy study. Diabetes Metab J 2011, 35:26-33.

22. Rong W, Cai LS: Comparing the efficacy of glimepiride and metformin in patients who are secondary sulfonylurea failure. Chinese J Diabetes 2004, 12:211-212.

23. Wang $K L$, Lei GD, Tang XX, Huang $Y H$ : Comparison of the efficacy of glimepiride and metformin in patients who are secondary sulfonylurea failure. Drug Research 2009, 18

24. UK Prospective Diabetes Study. http://www.dtu.ox.ac.uk/ukpds_trial/index.php.

25. HMR's Amaryl Launched For Diabetes In The USA. http://www. thepharmaletter.com/file/69437/hmrs-amaryl-launched-for-diabetes-in-theusa.html.

26. Schramm TK, Gislason GH, Vaag A, Rasmussen JN, Folke F, Hansen ML, Fosbol EL, Kober L, Norgaard ML, Madsen M, Hansen PR, Torp-Pedersen C: Mortality and cardiovascular risk associated with different insulin secretagogues compared with metformin in type 2 diabetes, with or without a previous myocardial infarction: a nationwide study. Eur Heart J 2011, 32:1900-1908

27. Pantalone KM, Kattan MW, Yu C, Wells BJ, Arrigain S, Jain A, Atreja A, Zimmerman RS: Increase in overall mortality risk in patients with type 2 diabetes receiving glipizide, glyburide or glimepiride monotherapy versus metformin: a retrospective analysis. Diabetes Obes Metabol 2012, 14:803-809.

28. Food and Drug Administration (FDA): Appoved drug products with therapeutic equivalence evaluations 32nd. U.S: Department of Health and Human Services, Food and Drug Administration, Center for Drug Evaluation and Research, Office of Pharmaceutical Science, and Office of Generic Drugs; 2012.

29. Higgins JPT, Green S (Eds): Cochrane handbook for systematic reviews of interventions version 5.1.0 [updated March 2011]. The Cochrane Collaboration; 2011. Available from www.cochrane-handbook.org.

30. Review Manager (RevMan): [Computer program]. Version 5.2. Copenhagen: The Nordic Cochrane Centre, The Cochrane Collaboration; 2012.

31. Viechtbauer $\mathrm{W}$ : Conducting meta-analyses in $\mathrm{R}$ with the metafor package. J Stat Software 2010, 36:1-48

32. The R Project for Statistical Computing. [http://www.r-project.org/] Accessed in 2013-06-19.

33. Ling ZM: The clinical observation of glimepiride in treating type 2 diabetes. J Clin Med Pract 2003, 7:574-575.

34. Ramachandran A, Snehalatha C, Salini J, Vijay V: Use of glimepiride and insulin sensitizers in the treatment of type 2 diabetes-a study in indians. J Assoc Physicians India 2004, 52:459-463.

35. Derosa G, Franzetti I, Gadaleta G, Ciccarelli L, Fogari R: Metabolic variations with oral antidiabetic drugs in patients with Type 2 diabetes: Comparison between glimepiride and metformin. Dia Nutr Metab 2004 17:143-150

36. González-Ortiz M, Martínez-Abundis E, Grupo para el Tratamiento de la Diabetes Mellitus con Combinaciones: Efficacy and safety of glimepiride plus metformin in a single presentation, as combined therapy, in patients with type 2 diabetes mellitus and secondary failure to glibenclamide, as monotherapy. Rev Inest Clin 2004, 56:327-333.

37. Tang JZ, Mao JP, Yang ZF, Zhou ZG, Tang WP, Feng Q: Effects of glimepiride and metformin on free fatty acid in patients with type 2 diabetes mellitus. J Cent South Univ Technol 2004, 29:631-634.

38. Ning J, Tang WL, Zhou ZG, Feng Q, Liu YH, Zhou WD: Effect of glimepiride and metformine on insulin resistance in type 2 diabetics. China J Mod Med 2006, 16:2224-2227.

39. Wu PS: The clinical efficacy of glimepiride on previously untreated type 2 diabetes patients. Appl J Gen Pract 2007, 5:1084-1085.

40. $\mathrm{Xu} \mathrm{ZQ,} \mathrm{Yu} \mathrm{JB:} \mathrm{Clinical} \mathrm{observation} \mathrm{of} \mathrm{glimepiride} \mathrm{and} \mathrm{metformin} \mathrm{in} \mathrm{newly}$ diagnosed type 2 diabetes. Chin J Misdiagn 2007, 7:2454-2455.

41. Li F, Tang GF, Tang TC, Yang YH, Zhang ZM: Glimepiride and metformin in the treatment of metabolism syndrome. J Bengbu Med Coll 2007, 32:445-446.

42. Rahman IU, Malik SA, Bashir M, Khan RU, Idrees M: Monotherapy with metformin or glimepiride and changes in serum sialic acid in type 2 diabetes mellitus. British J Diabetes Vascular Med 2011, 11:137-140.

43. Wang $\mathrm{H}$ : Observation of 68 cases of obese type 2 diabetes who are treated with metformin. Med Forum Mag 2011, 32:156-157.

44. WHO expert consultation: Appropriate body-mass index for Asian populations and its implications for policy and intervention strategies. Lancet 2004, 363:157-163.

45. Begg CB, Mazumdar M: Operating characteristics of a rank correlation test for publication bias. Biometrics 1994, 50:1088-1101.

46. Egger M, Smith GD, Schneider M, Minder C: Bias in meta-analysis detected by a simple, graphical test. BMJ 1997, 315:629-634.

47. Holstein A, Plaschke A, Egberts EH: Lower incidence of severe hypoglycaemia in patients with type 2 diabetes treated with glimepiride versus glibenclamide. Diabetes Metab Res Rev 2001, 17:467-473.

48. Sato J, Ohsawa I, Oshida Y, Fukuharu M, Hu XC, Tamagawa T, Sato Y: Comparison of the effects of three sulfonylureas on in vivo insulin action. Arzneimittel-Forsch 2001, 51:459-464.

49. Nathan DM, Buse JB, Davidson MB, Ferrannini E, Holman RR, Sherwin R, Zinmann B: Medical management of hyperglycemia in type 2 diabetes: a consensus algorithm for the initiation and adjustment of therapy: a consensus statement of the American Diabetes Association and the European Association for the Study of Diabetes. Diabetes Care 2009, 32:193-203.

50. Okayasu S, Kitaichi K, Hori A, Suwa T, Horikawa Y, Yamamoto M, Takeda J, Itoha $Y$ : The evaluation of risk factors associated with adverse drug reactions by metformin in type 2 diabetes mellitus. Biol Pharm Bull 2012 35:933-937.

51. Bennett WL, Wilson LM, Bolen S, Maruthur N, Singh S, Chatterjee R, Marinopoulos SS, Puhan MA, Ranasinghe P, Nicholson WK, Block L, Odelola O, Dalal DS, Ogbeche GE, Chandrasekhar A, Hutfless S, Bass EB, Segal JB: Oral diabetes medications for adults with type 2 diabetes: an update. In Comparative Effectiveness Review No 27 (Prepared by Johns Hopkins University Evidence-based Practice Center under Contract No 290-02-0018) AHRQ Publication No. 11-EHC038-EF. Edited by Rockville MD. Agency for Healthcare Research and Quality; 2011. Available at: http://www.ncbi.nlm. nih.gov/books/NBK55754/.

52. Bugos C, Austin M, Atherton T, Viereck C: Long-term treatment of type 2 diabetes mellitus with glimepiride is weight neutral: a meta-analysis. Diabetes Res Clin Pract 2000, 50:251-254.

53. Garber AJ, Duncan TG, Goodman AM, Mills DJ, Rohlf JL: Efficacy of metformin in type II diabetes: results of a double-blind, placebo-controlled, dose-response trial. Am J Med 1997, 102:491-497.

54. ClinicalTrials.gov. http://clinicaltrials.gov/ct2/results?term=Diabetes +metformin+Glimepiride

doi:10.1186/1758-5996-5-70

Cite this article as: Zhu et al:: Comparative efficacy of glimepiride and metformin in monotherapy of type 2 diabetes mellitus: meta-analysis of randomized controlled trials. Diabetology \& Metabolic Syndrome 2013 5:70. 